\title{
Systemic features of retinal vasculopathy with cerebral leukoencephalopathy and systemic manifestations: a monogenic small vessel disease
}

\author{
N. Pelzer ${ }^{1}$, E. S. Hoogeveen ${ }^{2}$, J. Haan ${ }^{1,3}$, R. Bunnik ${ }^{1}$, C. C. Poot ${ }^{1}$, E. W. van Zwet ${ }^{4}$, A. Inderson ${ }^{5}$, A. J. Fogteloo ${ }^{6}$, \\ M. E. J. Reinders ${ }^{7}$, H. A. M. Middelkoop ${ }^{1,8}$, M. C. Kruit ${ }^{2}$, A. M. J. M. van den Maagdenberg ${ }^{1,9},{ }^{2}$. D. Ferrari ${ }^{1}$ \\ \& G. M. Terwindt ${ }^{1}$ \\ From the ${ }^{1}$ Department of Neurology; ${ }^{2}$ Department of Radiology, Leiden University Medical Centre, Leiden; ${ }^{3}$ Department of Neurology, Alrijne \\ Hospital, Leiderdorp; ${ }^{4}$ Department of Biomedical Data Sciences; ${ }^{5}$ Department of Gastroenterology-Hepatology; ${ }^{6}$ Department of Internal \\ Medicine (Acute Care); ${ }^{7}$ Department of Internal Medicine (Nephrology), Leiden University Medical Centre; ${ }^{8}$ Institute of Psychology, Health, \\ Medical and Neuropsychology Unit, Leiden University; and ${ }^{9}$ Department of Human Genetics, Leiden University Medical Centre, Leiden, The \\ Netherlands
}

Abstract. Pelzer N, Hoogeveen ES, Haan J, Bunnik R, Poot CC, van Zwet EW, Inderson A, Fogteloo AJ, Reinders MEJ, Middelkoop HAM, Kruit MC, van den Maagdenberg AMJM, Ferrari MD, Terwindt GM (Leiden University Medical Centre, Leiden; Alrijne Hospital, Leiderdorp; Leiden University, Leiden, The Netherlands). Systemic features of retinal vasculopathy with cerebral leukoencephalopathy and systemic manifestations: a monogenic small vessel disease. J Intern Med 2019; 285: 317-332.

Background. Retinal vasculopathy with cerebral leukoencephalopathy and systemic manifestations (RVCL-S) is a small vessel disease caused by C-terminal truncating TREX1 mutations. The disease is typically characterized by vascular retinopathy and focal and global brain dysfunction. Systemic manifestations have also been reported but not yet systematically investigated.

Methods. In a cross-sectional study, we compared the clinical characteristics of 33 TREX 1 mutation carriers $(\mathrm{MC}+)$ from three Dutch RVCL-S families with those of 37 family members without TREX1 mutation (MC-). All participants were investigated using personal interviews, questionnaires, physical, neurological and neuropsychological examinations, blood and urine tests, and brain MRI.

Results. In MC+, vascular retinopathy and Raynaud's phenomenon were the earliest symptoms presenting from age 20 onwards. Kidney disease became manifest from around age 35, followed by liver disease, anaemia, markers of inflammation and, in some $\mathrm{MC}+$, migraine and subclinical hypothyroidism, all from age 40. Cerebral deficits usually started mildly around age 50, associated with white matter and intracerebral mass lesions, and becoming severe around age 60-65.

Conclusions. Retinal vasculopathy with cerebral leukoencephalopathy and systemic manifestations is a rare, but likely underdiagnosed, systemic small vessel disease typically starting with vascular retinopathy, followed by multiple internal organ disease, progressive brain dysfunction, and ultimately premature death.

Keywords: kidney disease, liver disease, microangiopathy, neurology, Raynaud's phenomenon, thyroid disease.

\section{Introduction}

Retinal vasculopathy with cerebral leukoencephalopathy and systemic manifestations (RVCL-S) is an autosomal dominant neurovascular syndrome caused by heterozygous C-terminal frameshift mutations in TREX1 and ultimately leading to premature death $[1,2]$. Before the gene was identified, the disease has been known as cerebroretinal vasculopathy (CRV) [3], hereditary vascular retinopathy (HVR) [4], hereditary endotheliopathy, retinopathy, nephropathy and stroke (HERNS) [5] and hereditary systemic angiopathy (HSA) [6]. The best-known features of RVCL-S are progressive blindness due to vascular retinopathy, neurological manifestations of focal and diffuse brain dysfunction, and white matter and intracerebral mass lesions on neuroimaging [2]. As 
observed in our (outpatient) clinic, complications of general debilitation due to vascular dementia and cerebral deficits, for example aspiration pneumonia, are the main cause of premature death in RVCL-S. Less well described are systemic manifestations such as liver and kidney disease, anaemia, hypertension and Raynaud's phenomenon [2]. In some cases, kidney failure was severe and contributed to a patient's premature death. As these systemic features may precede the characteristic ophthalmological and neurological features of RVCL-S, establishing a correct diagnosis can be challenging [2]. To improve early recognition, diagnosis and treatment of RVCL-S and, in particular, its systemic manifestations, we conducted a crosssectional study, aimed at better characterizing the extracranial manifestations of RVCL-S. To this end, we carefully investigated 33 symptomatic and asymptomatic RVCL-S TREX1 mutation carriers $(\mathrm{MC}+)$ and 37 nonmutation carriers ( $\mathrm{MC}-$ ) from three Dutch RVCL-S families, using a standardized protocol specifically focussing on systemic signs and symptoms of the disease.

\section{Materials and methods}

\section{Participants and genetic screening}

We invited all established $\mathrm{MC}+$ and their 1 st and 2 nd degree family members aged $\geq 18$ years from three known (but unrelated) Dutch RVCL-S families, regardless of MC status. Consenting family members with unknown MC status were screened for TREX1 mutations using genomic DNA extracted from peripheral leucocytes, PCR and direct Sanger sequencing as described before [1]. As RVCL-S cannot yet be treated, participants could opt to remain unaware of the genetic test results. Family members without a TREX1 mutation were included as controls. Matching by age and gender was not possible due to the limited number of available family members. The study was approved by the Medical Ethics Committee of LUMC. All participants provided written informed consent prior to inclusion.

\section{Study design}

This was a cross-sectional study that, for logistic reasons, consisted of three standardized visits (Figure 1). In visit 1, participants were interviewed to assess disease features and blood was sampled for DNA isolation. Raynaud's phenomenon was diagnosed using the questionnaire by Miller et al. [7] (cut-off score $>4$ ) and the novel international consensus criteria (except for use of photographs, which were not available) [8]. A lifetime migraine diagnosis, including its subtypes, was established according to the International Classification of Headache Disorders (ICHD) criteria [9], using a validated migraine questionnaire [10]. Depression was established with a score of $\geq 8$ on the Hospital Anxiety and Depression Scale (HADS-D) [11] and/ or a score of $\geq 16$ on the Centre for Epidemiological Studies Depression Scale (CES-D) [12], and anxiety with a HADS-A score of $\geq 8$ [13]. Physical and cognitive complaints, medical history, medication use, lifestyle habits and socio-demographic characteristics were assessed during a semistructured interview.

Most family members with unknown MC status declined to know their diagnosis. Finding evidence of vascular retinopathy in a person from a family with RVCL-S would almost certain confirm the diagnosis. Not disclosing the results of an ophthalmological examination was however not an option as retinopathy can potentially be treated. We therefore decided not to screen for retinopathy in family members with unknown MC status. We did advise them, though, to consult an ophthalmologist as soon as they would get visual complaints. Information on signs of retinopathy in this study thus came solely from reports of treating ophthalmologists of established MC+.

In visit 2, physical examination was performed. Blood pressure was measured on each arm, in sitting position, using the same electronic oscillometric device with a cuff around the upper arm. Hypertension was defined as: (i) use of antihypertensive medication; (ii) systolic blood pressure $>140 \mathrm{mmHg}$; or (iii) diastolic blood pressure $>90 \mathrm{mmHg}$ [14]. Height and weight were measured to calculate body mass index (BMI) and a structured neurological examination was performed. To assess general disability, all participants were rated according to the modified Rankin Scale (mRS) and the Barthel index of activities of daily living [15, 16]. Blood and urine samples were collected in the morning, after at least $8 \mathrm{~h}$ of fasting (median $12 \mathrm{~h}$, range 8-17) and were transported immediately for laboratory assays according to clinical protocols. Kidney disease was defined as glomerular filtration

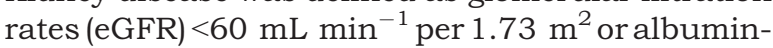
creatinine ratio or $>3 \mu \mathrm{g} \mu \mathrm{mol}^{-1}$.

In visit 3, all participants first underwent a neuropsychological examination in the afternoon. 


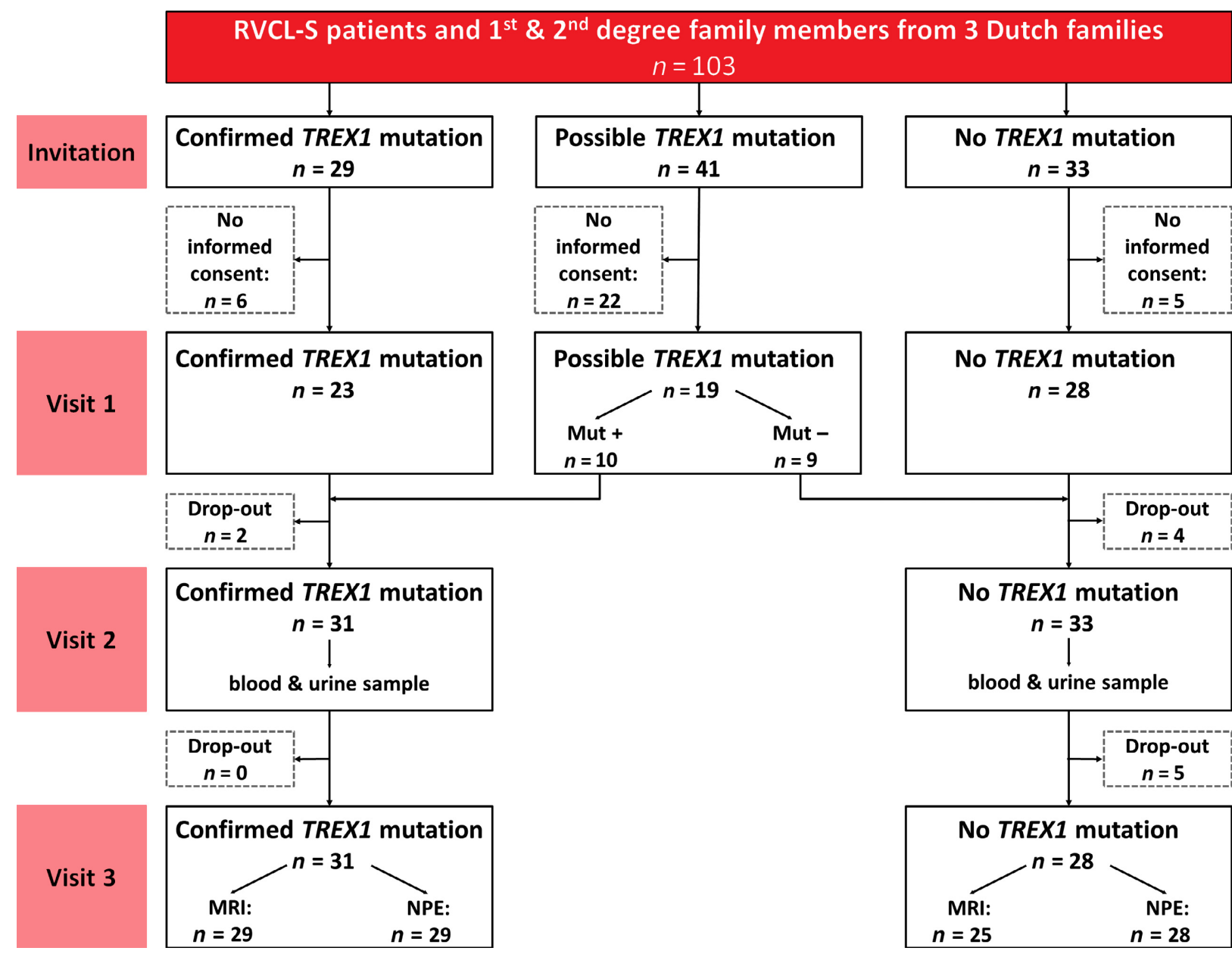

Fig. 1 Flowchart depicting inclusion of participants from the three Dutch RVCL-S families into the study. Dropouts after visit 1 (interview and questionnaires) and visit 2 (sampling and physical examination) are shown. Six participants (including two $M C+$ ) dropped out after visit 1. Five family members considered multiple visits too much of a burden and one subject was physically unable to visit the LUMC. For visit 2, the number of physical examinations, urine samples, and vena punctures for blood sampling are depicted. For visit 3, the number of brain MRIs and neuropsychological examinations (NPE) are shown. An additional five participants (all MC-) could not attend visit 3 because of lack of time. Two MC+ could not complete the neuropsychological examination because of poor vision and anarthria. Two other MC+ and three $M C-$ withdrew from or had a relative contra-indication for brain MRI.

Examinations included the Wechsler memory scale-fourth edition (WMS-IV) [17] and the Cambridge Cognitive Examination-Revised (CAMCOG$\mathrm{R})$. This assesses orientation, language, memory, attention, praxis, calculation, abstraction and perception [18] and includes the Mini-Mental State Examination (MMSE) [19]. In the evening, a brain MRI was performed. Volume of brain white matter hyperintensities was assessed on FLAIR images (see for methods legend of Table 5).

\section{Statistical analysis}

Variables were reported as medians [interquartile range $(\mathrm{IQR})]$ or percentages. For continuous variables without a normal distribution in our population Mann-Whitney $U$-tests were applied. Categorical variables were compared with Pearson Chi-Square tests, or Fisher's Exact tests when appropriate, and by calculating relative risks (RR). Corrections for multiple testing were not applied, 
as analyses were meant to be exploratory and hypothesis-generating. Results with $P<0.05$ were considered statistically significant. All statistics were performed using sPSS 23.0 (IBM Corp., Armonk, NY, USA).

\section{Results}

Genetic testing and socio-demographic characteristics of the study population

In total 103 members of three Dutch RVCL-S families were invited to participate (Figure 1). Thirty-three (32\%) declined to participate, because: (i) participation was considered too much of a burden ( $n=21)$; (ii) they did not want to participate in any study on RVCL-S $(n=6)$; (iii) health issues precluding visiting the hospital $(n=4$ : one $\mathrm{MC}+$ due to RVCL-S-related poor vision; three unknown); and (iv) living abroad $(n=2)$. Of 11 nonparticipants the MC status (6 MC+; $5 \mathrm{MC}-$ ) and clinical details were known from previous studies. Their clinical status did not appear to be different from participants. The remaining 22 nonparticipants had a $50 \%(n=20)$ or $25 \%(n=2)$ a priori chance of carrying a TREX1 mutation. Their clinical status was unknown, but most $(17 / 22=77 \%)$ were younger than 40 years of age. Overall, nonparticipants were younger than participants (median age 34 vs. 46 years; $P=0.02$ ) and more often male $(25 / 33=76 \%$ vs. $30 / 70=43 \% ; P=0.002)$.

In total $37 \mathrm{MC}-$ and $33 \mathrm{MC}+(23$ previously known, 10 newly detected) participated in the study. MC+ and $\mathrm{MC}-$ did not differ with respect to age, gender, education level, cigarette pack years, or alcohol use, but $\mathrm{MC}+$ used less caffeine than $\mathrm{MC}-$ (Table 1). With respect to medication use, statins were only used by $\mathrm{MC}+$ and $\mathrm{MC}+$ used more antihypertensive drugs. Seven $\mathrm{MC}+$ (median age 57 ; range 52-65 years) (but none of $\mathrm{MC}-$ ) were unfit to work, in particular because they were too slow in performing complex tasks. This impairment was unrelated to visual impairment. A summary of all symptoms of RVCL-S is provided in Figure 2.

\section{Association with vascular retinopathy}

All known $23 \mathrm{MC}+$ had signs of vascular retinopathy, of which 15 (65\%; median age 57 years) had undergone (pan)retinal laser photocoagulation. In six (median age 41 years), retinopathy was mild not yet requiring treatment. Thirteen experienced visual field defects (12 after retinal laser photocoagulation). In order to remain unaware of their mutation status, $10 \mathrm{MC}+$ (median age 24 years), who were identified during the study, were not investigated by an ophthalmologist. They did not have visual complaints.

\section{Association with internal organ disease}

Internal organ disease was investigated at three levels: (i) subjective complaints (Table 2; $33 \mathrm{MC}+$ and $37 \mathrm{MC}-$ ); (ii) objective signs at physical examination (Table 3; $32 \mathrm{MC}+$ and $32 \mathrm{MC}-$ ); and (iii) laboratory tests (Figure 3 and Table 4; $31 \mathrm{MC}+$ and 33 MC-).

Kidney disease was found in 36\% of MC+ (Table 2). Cystitis, pyelonephritis, nephrolithiasis and haematuria occurred equally frequent in $\mathrm{MC}+$ and MC-. Urine albumin concentrations [median (IQR) 30 (10-96) $\mathrm{mg} \mathrm{mL}^{-1}$ ] and microalbumin/creatinine ratios [median (IQR) $6.1 \quad(1.5-13.4)$ $\mu \mathrm{g} \mu \mathrm{mol}^{-1}$ ] were increased in MC+ (Table 4). Albuminuria was not explained by concurrent hypertension (Figure S1). Haematuria was not routinely assessed, but absent in 24-h urine of nine MC+.

Liver disease, usually asymptomatic, was found in $27 \%$ of MC+ (Table 2). Five MC+ had a history of jaundice, four at a young age with jaundice simultaneously in relatives in three (i.e. highly suggestive of a shared infectious cause), and one in association with cholelithiasis. Gamma-glutamyl transferase $(\gamma-\mathrm{GT})$, alkaline phosphatase (ALP) and aspartate aminotransferase (ASAT) were increased in $\mathrm{MC}+$ (Table 4). Most MC+ with elevated liver enzymes share a same pattern of ALP and $\gamma$ GT $2-3 x$ ULN (upper limit of normal) and normal to minimally elevated aminotransferase (1-1,5x ULN) and normal bilirubin levels. None had primary liver disease. One $\mathrm{MC}+$ had cryptogenic liver cirrhosis (all other causes of cirrhosis were excluded) and one had hepatic steatosis. Previous liver ultrasound examination did not show abnormalities in six other MC+ with increased $\gamma$-GT levels.

A history of anaemia was frequently reported by $\mathrm{MC}+(33 \%)$, but also by $\mathrm{MC}-(19 \% ; P=0.17)$ (Table 2). Laboratory results clearly showed lower haemoglobin and haematocrit levels and slightly higher mean corpuscular volumes (MCV) in $\mathrm{MC}+$ (Table 4). One MC+ used iron supplements, another darbepoetin (for severe kidney dysfunction), and one $\mathrm{MC}+$ just had finished treatment with iron supplements. Colonoscopy results were available for four $\mathrm{MC}+$; all had signs of intestinal 


\section{Systemic features of RVCL-S / N. Pelzer et al.}

Table 1 Demographics of RVCL-S family members with (MC+) and without (MC-) a TREX1 mutation

\begin{tabular}{|c|c|c|c|}
\hline & $\mathrm{MC}+(n=33)$ & $\mathrm{MC}-(n=37)$ & $P$-value \\
\hline \multicolumn{4}{|l|}{ Age (years) } \\
\hline Median (IQR) & $51.9(28.6-56.0)$ & $45.2(38.8-61.9)$ & ns \\
\hline$\geq 40$ years, $n(\%)$ & $20(61)$ & $28(76)$ & ns \\
\hline \multicolumn{4}{|l|}{ Sex } \\
\hline Male, $n(\%)$ & $14(42)$ & $16(43)$ & $\mathrm{ns}$ \\
\hline Males $\geq 40$ years, $n(\%)$ & $6(30)$ & $13(46)$ & ns \\
\hline \multicolumn{4}{|l|}{ Pedigree and TREX1 mutation } \\
\hline A: p.Val235 fs, $n(\%)$ & $21(64)$ & $33(89)$ & \\
\hline B: p.Val235 fs, $n(\%)$ & $7(21)$ & $1(3)$ & \\
\hline C: p.Leu287 fs, $n(\%)$ & $5(15)$ & $3(8)$ & \\
\hline \multicolumn{4}{|l|}{ Smoking } \\
\hline Present, $n(\%)$ & $5(15)$ & $2(5)$ & ns \\
\hline Past, $n(\%)$ & $11(33)$ & $12(32)$ & \\
\hline Never, $n(\%)$ & $17(52)$ & $23(62)$ & \\
\hline Median pack years (IQR) & $0(0-2)$ & $0(0-6)$ & $\mathrm{ns}$ \\
\hline \multicolumn{4}{|l|}{ Alcohol use } \\
\hline Median (IQR) (u per wk) & $2(0-9)$ & $3(0-5)$ & ns \\
\hline \multicolumn{4}{|l|}{ Caffeine use } \\
\hline Median (IQR) (u per day) & $5(3-7)$ & 7 (4-9) & $P=0.02$ \\
\hline \multicolumn{4}{|l|}{ Medication use } \\
\hline Migraine prophylactics, $n(\%)$ & $1(3)$ & $0(0)$ & - \\
\hline Antihypertensive drugs, $n(\%)$ & $8(24)$ & $4(11)$ & ns \\
\hline Statins, $n(\%)$ & $4(12)$ & $0(0)$ & - \\
\hline Thrombocyte aggregation inhibitors, $n(\%)$ & $5(15)$ & $3(8)$ & ns \\
\hline Anticoagulants, $n(\%)$ & $0(0)$ & $1(3)$ & - \\
\hline Antidepressants, $n(\%)$ & $3(9)$ & $1(3)$ & ns \\
\hline \multicolumn{4}{|l|}{ Education level } \\
\hline Median (IQR) & $4(3-5)$ & $4(3-6)$ & ns \\
\hline 1. Primary school, $n(\%)$ & $0(0)$ & $1(3)$ & \\
\hline 2. Secondary 1 (low-level), $n(\%)$ & $4(12)$ & $8(22)$ & \\
\hline 3. Secondary 2 (medium-level), $n(\%)$ & $5(15)$ & $2(5)$ & \\
\hline 4. Secondary 3 (high-level), $n(\%)$ & $3(9)$ & $1(3)$ & \\
\hline 5. Tertiary 1 (low-level), $n$ (\%) & $14(42)$ & $10(27)$ & \\
\hline 6. Tertiary 2 (medium-level), $n(\%)$ & $5(15)$ & $15(41)$ & \\
\hline 7. Tertiary 3 (university), $n(\%)$ & $2(6)$ & $0(0)$ & \\
\hline
\end{tabular}

IQR, interquartile range; ns, not statistically significant; $u$, units.

angiodysplasia as a possible source of (gastro) intestinal bleeding. One patient underwent video capsule endoscopy, revealing numerous dot-sized bleeding foci throughout the small intestine. Of the nine subjects who reported incidental faecal blood loss, one $\mathrm{MC}+$ was anaemic (haemoglobin 6.2), but she had noticed (bright red) blood loss only once.
Of the assessed (cardio)vascular complaints only swollen ankles were more prevalent in $\mathrm{MC}+$ (Table 2). On physical examination, there were no differences in presence of varicose veins, ankle oedema or livedo reticularis (Table 3). Prevalence of hypertension was high in both $\mathrm{MC}+(39 \%)$ and MC- (35\%). Median systolic and diastolic blood 


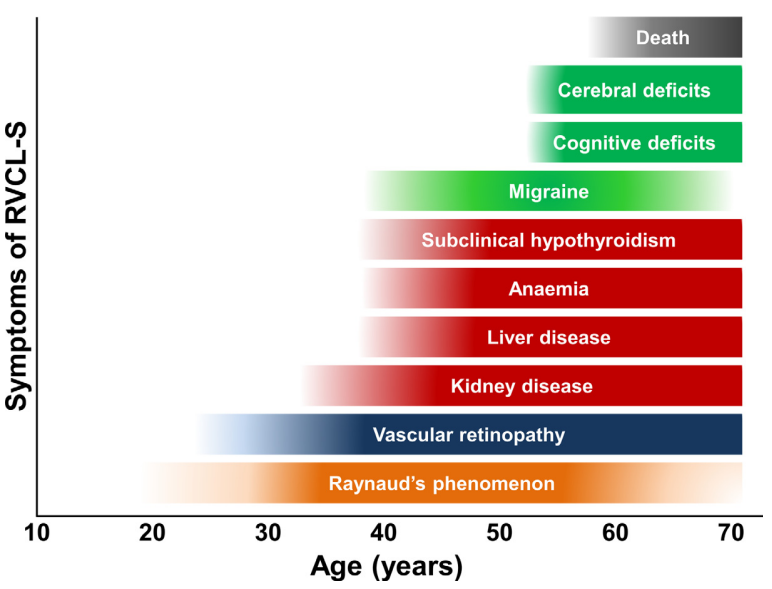

Fig. 2 Clinical course of RVCL-S. Symptoms of RVCL-S derived from previous studies [2] and the current crosssectional investigation of RVCL-S patients laged 1865 years). Vascular retinopathy and Raynaud's phenomenon were the earliest symptoms presenting from age 20 onwards. Kidney disease became manifest from around age 35, followed by liver disease, anaemia, and, in some $\mathrm{MC}+$, migraine and subclinical hypothyroidism, all from age 40. Cerebral and cognitive deficits usually started mildly around age 50, associated with increasing volume of white matter hyperintensities and intracerebral mass lesions, and becoming severe and ultimately lethal around age 60-65.

pressures measured in visit 2 (with standardized conditions) were virtually the same in $\mathrm{MC}+$ and MC- (Table 3). Only the eldest $\mathrm{MC}+$ reported coronary artery disease. There were no reports of (hypertensive) cardiomyopathy in $\mathrm{MC}+$, which was reported previously in RVCL-S [6, 20, 21].

A novel finding in RVCL-S were increased thyroid stimulating hormone (TSH) levels with normal free thyroxine (fT4) levels, indicating subclinical hypothyroidism (Table 4). One MC+ had been treated for presumed Graves' disease, but had normal TSH and fT4 levels while using levothyroxine.

There were no signs of rheumatic disease (e.g. joint pains or inflammation), autonomic dysfunction (e.g. orthostatic hypotension, urine incontinence or erectile dysfunction), or avascular necrosis of the femur head [6]. Previous ultrastructural studies revealed abnormalities in skin samples of RVCL-S patients [5], but besides livedo reticularis, varicose veins and ankle oedema (Table 3 ), which were not more prevalent in $\mathrm{MC}+$, no skin abnormalities were found during physical examination in $\mathrm{MC}+$. With regard to common vascular risk factors: median BMI $(P=0.31)$ and cholesterol levels $(P=0.91)$ did not differ between $\mathrm{MC}+$ and MC- (Table 3), with only four MC+ taking statins. Fasting glucose and $\mathrm{HbA} 1 \mathrm{c}$ values were normal in all MC+, ruling out diabetes mellitus. Several markers of inflammation and coagulation (erythrocyte-sedimentation rates (ESR), fibrinogen, Ddimer concentrations, and prothrombin time (PT)) were increased in $\mathrm{MC}+$, other coagulation markers were not abnormal in $\mathrm{MC}+$.

\section{Correlating internal organ disease with age}

By plotting laboratory parameters against age, we constructed a pseudolongitudinal course of disease (Figures 2 and 3; Table 4). Kidney disease (increased urine albumin and microalbumin-creatinine ratios) were abnormal already before age 40, while all other internal organ disease developed from age 40 onwards (Figure 2 and 3).

\section{Association with Raynaud's phenomenon}

The prevalence of Raynaud's phenomenon was increased among MC+ according to Miller's criteria $[R R(95 \% \mathrm{CI})=1.90(1.15-3.13)]$ and recent international consensus criteria $[\mathrm{RR}(95 \% \mathrm{CI})=2.24$ (1.03-4.88)] (Table 2) [7, 8]. Onset was mostly before age $20 \quad(10 / 20=50 \% \mathrm{MC}+$ and $7 /$ $12=58 \% \mathrm{MC}-$ according to Miller's criteria; 7/ $14=50 \% \mathrm{MC}+$ and $5 / 7=71 \% \mathrm{MC}-$ according to the international consensus criteria). Symptoms were mostly mild, and there were no ischaemic injuries.

\section{Neurological and cognitive features}

$\mathrm{MC}+$ more often had subjective memory loss and focal neurological deficits. Notable neurological deficits were only found in $\mathrm{MC}+$ older than 50 years.

Lifetime prevalence of migraine was similar in $\mathrm{MC}+$ $(27 \%)$ and $\mathrm{MC}-(38 \%) ; \quad P=0.35 ; \quad \mathrm{RR} \quad(95 \%$ CI $=0.72 \quad(0.36-1.42)$. Aura symptoms were reported by $6 / 9(67 \%)$ of $\mathrm{MC}+$ and $12 / 14(86 \%)$ of $\mathrm{MC}-$ with migraine. There was a trend for migraine starting at a later age in $\mathrm{MC}+[$ median $(\mathrm{IQR})=40$ (15-43) years] vs. MC- [17 (8-20) years; $P=0.07]$, which reached significance for migraine with aura $[$ median $(\mathrm{IQR})=40(30-47)]$ years in $\mathrm{MC}+$ versus $\mathrm{MC}-[$ median $(\mathrm{IQR})=20(19-22)$ years; $P=0.01]$. 


\section{Systemic features of RVCL-S / N. Pelzer et al.}

Table 2 Complaints or self-reported diagnoses in members with (MC+) and without (MC-) a TREX1 mutation of RVCL-S families

\begin{tabular}{|c|c|c|c|c|}
\hline & $\begin{array}{l}\mathrm{MC}+ \\
(n=33)\end{array}$ & $\begin{array}{l}\mathrm{MC}- \\
(n=37)\end{array}$ & $\begin{array}{l}\text { Statistical } \\
\text { analysis } \chi^{2}\end{array}$ & $\begin{array}{l}\text { Statistical analysis } \\
\text { Risk Ratio (95\% CI) }\end{array}$ \\
\hline \multicolumn{5}{|l|}{ Kidneys and urinary tract symptoms } \\
\hline Kidney disease, $n(\%)$ & $12(36)$ & $1(3)$ & $P<0.001$ & \multirow[t]{7}{*}{$13.5(1.8-98.0)$} \\
\hline Frequent cystitis, $n(\%)$ & $4(12)$ & $6(16)$ & ns & \\
\hline Pyelonephritis, $n(\%)$ & $2(6)$ & $2(5)$ & ns & \\
\hline Kidney stones, $n(\%)$ & $3(9)$ & $4(11)$ & ns & \\
\hline Haematuria, $n(\%)$ & $3(9)$ & $4(11)$ & ns & \\
\hline Urine urge incontinence, $n(\%)$ & $5(16)$ & $4(11)$ & ns & \\
\hline Urine stress incontinence, $n(\%)$ & $1(3)$ & $5(14)$ & ns & \\
\hline \multicolumn{5}{|l|}{ Hepatic and gastrointestinal symptoms } \\
\hline Liver disease, $n(\%)$ & $9(27)$ & $2(5)$ & $P=0.01$ & \multirow[t]{6}{*}{$5.0(1.2-21.7)$} \\
\hline Diarrhoea, $n(\%)$ & $6(18)$ & $2(5)$ & ns & \\
\hline Constipation, $n(\%)$ & $12(35)$ & $15(41)$ & ns & \\
\hline Faecal blood, $n(\%)$ & $5(15)$ & $4(11)$ & ns & \\
\hline Cholelithiasis, $n(\%)$ & $3(9)$ & $2(5)$ & ns & \\
\hline Jaundice, $n(\%)$ & $5(15)$ & $1(3)$ & ns & \\
\hline \multicolumn{5}{|l|}{ Cardiovascular symptoms } \\
\hline Orthostatic hypotension, $n(\%)$ & $15(47)$ & $22(60)$ & ns & \multirow{10}{*}{$4.5(1.7-12.1)$} \\
\hline Fatigue, $n(\%)$ & $15(46)$ & $10(27)$ & ns & \\
\hline Anaemia, $n(\%)$ & $11(33)$ & 7 (19) & ns & \\
\hline Chest pains with exercise, $n(\%)$ & $2(6)$ & $3(8)$ & ns & \\
\hline Exercise-induced dyspnoea, $n(\%)$ & $9(28)$ & $4(11)$ & ns & \\
\hline Palpitations, $n(\%)$ & $16(49)$ & $13(35)$ & ns & \\
\hline Swollen ankles, $n(\%)$ & $16(49)$ & $4(11)$ & $P<0.001$ & \\
\hline Varicose veins, $n(\%)$ & $11(33)$ & $11(30)$ & ns & \\
\hline Intermittent claudication, $n(\%)$ & $3(9)$ & $1(3)$ & ns & \\
\hline Nocturia, $n(\%)$ & $8(24)$ & $6(16)$ & ns & \\
\hline \multicolumn{5}{|l|}{ Musculoskeletal symptoms } \\
\hline Joint pains, $n(\%)$ & $8(24)$ & $12(32)$ & ns & \\
\hline Joint inflammation, $n(\%)$ & $0(0)$ & $3(8)$ & ns & \\
\hline Raynaud's phenomenon ${ }^{\mathrm{a}}, n(\%)$ & $14(42)$ & 7 (19) & $P=0.03$ & $2.2(1.0-4.9)$ \\
\hline Migraine $^{\mathrm{b}}, n(\%)$ & $9(27)$ & $14(38)$ & ns & \\
\hline
\end{tabular}

ns, not statistically significant.

${ }^{a}$ According to the international consensus criteria from 2014 [8]. ${ }^{b}$ According to the International Classification of Headache Disorders (ICHD), third edition [9].

Except for a higher prevalence of depressive symptoms in $\mathrm{MC}+\geq 40$ years, neuropsychological tests did not reveal clear differences between $\mathrm{MC}+$ and MC- (Table 5). We found no major cognitive impairment in $\mathrm{MC}+$ [median (IQR) CAMCOG-R score of $93.0(89.0-96.0)]$ in $\mathrm{MC}+$ versus 93.5
(9.0-97.8) in MC-. Only the eldest MC+ fulfilled criteria for dementia.

The volume of white matter hyperintensities on brain MRI was increased in $\mathrm{MC}+\geq 40$ years (Table 5). While several $\mathrm{MC}+$ had suffered from 


\section{Systemic features of RVCL-S / N. Pelzer et al.}

Table 3 Results of general disability rating scales and physical examinations in RVCL-S family members with (MC+) and without (MC-) a TREX1 mutation

\begin{tabular}{|c|c|c|c|}
\hline & $\mathrm{MC}+$ & $\mathrm{MC}-$ & $P$-value \\
\hline Modified Rankin Scale (mRS), $n(\%)$ & $(n=32)$ & $(n=32)$ & $P=0.002$ \\
\hline 0 & $11(34)$ & $27(82)$ & \\
\hline 1 & $10(31)$ & $5(15)$ & \\
\hline 2 & $8(25)$ & $1(3)$ & \\
\hline 3 & $0(0)$ & $0(0)$ & \\
\hline 4 & $2(6)$ & $0(0)$ & \\
\hline 5 & $1(3)$ & $0(0)$ & \\
\hline \multicolumn{4}{|l|}{ Barthel index, $n(\%)$} \\
\hline 20 & $24(75)$ & $30(91)$ & ns \\
\hline 19 & $5(16)$ & $3(9)$ & \\
\hline 4 & $1(3)^{\mathrm{a}}$ & $0(0)$ & \\
\hline 3 & $1(3)^{\mathrm{a}}$ & $0(0)$ & \\
\hline 2 & $1(3)$ & $0(0)$ & \\
\hline Body mass index, measured & $(n=29)$ & $(n=33)$ & \\
\hline Median (IQR) $\left(\mathrm{kg} \mathrm{m}^{-2}\right)$ & $22.8(21.5-26.3)$ & $24.9(21.4-26.9)$ & ns \\
\hline Hypertension & $(n=29)$ & $(n=33)$ & \\
\hline Present, $n(\%)$ & $13(39)$ & $13(35)$ & ns \\
\hline Antihypertensive drugs, $n(\%)$ & $8(24)$ & $4(11)$ & \\
\hline $\begin{array}{l}\text { Median systolic (IQR)/diastolic (IQR) blood } \\
\text { pressure }(\mathrm{mmHg}) \text {, visit } 1\end{array}$ & $132(120-154) / 85(74-98)$ & $128(121-146) / 84(80-94)$ & \\
\hline $\begin{array}{l}\text { Median systolic (IQR)/diastolic (IQR) blood } \\
\text { pressure }(\mathrm{mmHg}) \text {, visit } 2\end{array}$ & $129(111-144) / 84(72-94)$ & $128(117-139) / 86$ (81-95) & \\
\hline Varicose veins & $(n=29)$ & $(n=33)$ & \\
\hline Present, $n(\%)$ & $23(72)$ & $23(70)$ & ns \\
\hline Mild, $n$ & 14 & 18 & ns \\
\hline Moderate, $n$ & 5 & 0 & \\
\hline Severe (corona phlebectatica), $n$ & 4 & 5 & \\
\hline Ankle oedema & $(n=29)$ & $(n=33)$ & \\
\hline Present, $n(\%)$ & $6(19)$ & $3(9)$ & ns \\
\hline Livedo reticularis & $(n=29)$ & $(n=33)$ & ns \\
\hline Present, $n(\%)$ & $7(22)$ & $8(24)$ & \\
\hline
\end{tabular}

IQR, interquartile range; ns, not statistically significant.

${ }^{a}$ Two subjects were also diagnosed with Multiple System Atrophy, unrelated to RVCL-S.

Fig. 3 Laboratory parameters in blood. Parameters were investigated to assess kidney function (estimated glomerular filtration rate (eGFR) and microalbumin-creatinin-ratio), liver function (gamma-glutamyl transpeptidase ( $\gamma$-GT) and alkaline phosphatase], thyroid function [thyroid stimulating hormone (TSH) and free thyroxine (T4)], blood count (haemoglobin), and inflammation [erythrocyte-sedimentation rate (ESR) and high sensitivity C-reactive protein (CRP)]. One blood glucose assay and one homocysteine assay failed, both in $M C+$. Symbols: Black circles and grey boxes: $M C+$; white circles and boxes: MC-; box plot whiskers: medians, lower and higher quartiles, lower and higher extreme values; $* \mathrm{P}<0.05 ; * * \mathrm{P}<0.001$. 

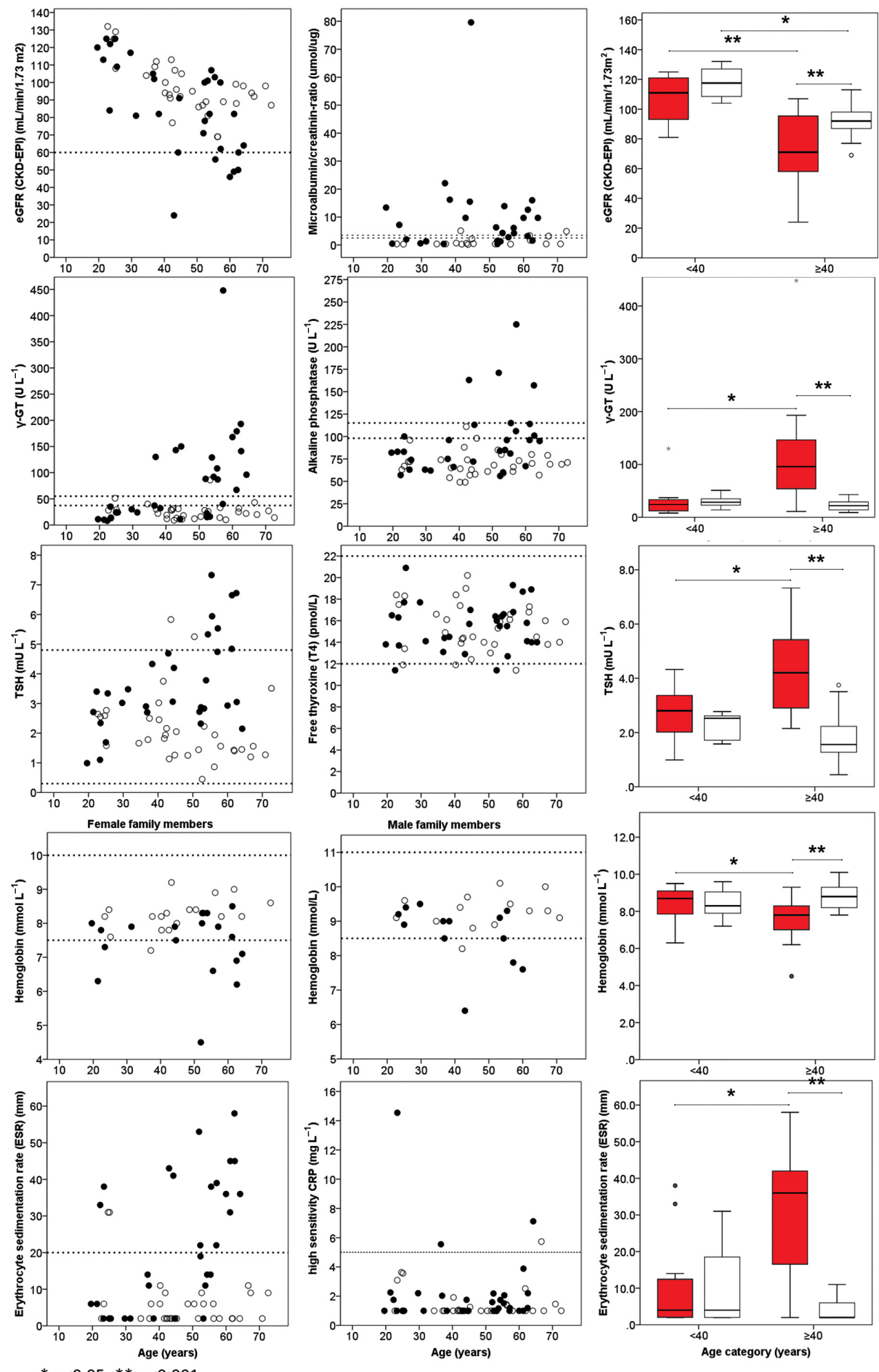

${ }^{*} p<0.05 ;{ }^{* *} p<0.001$ 

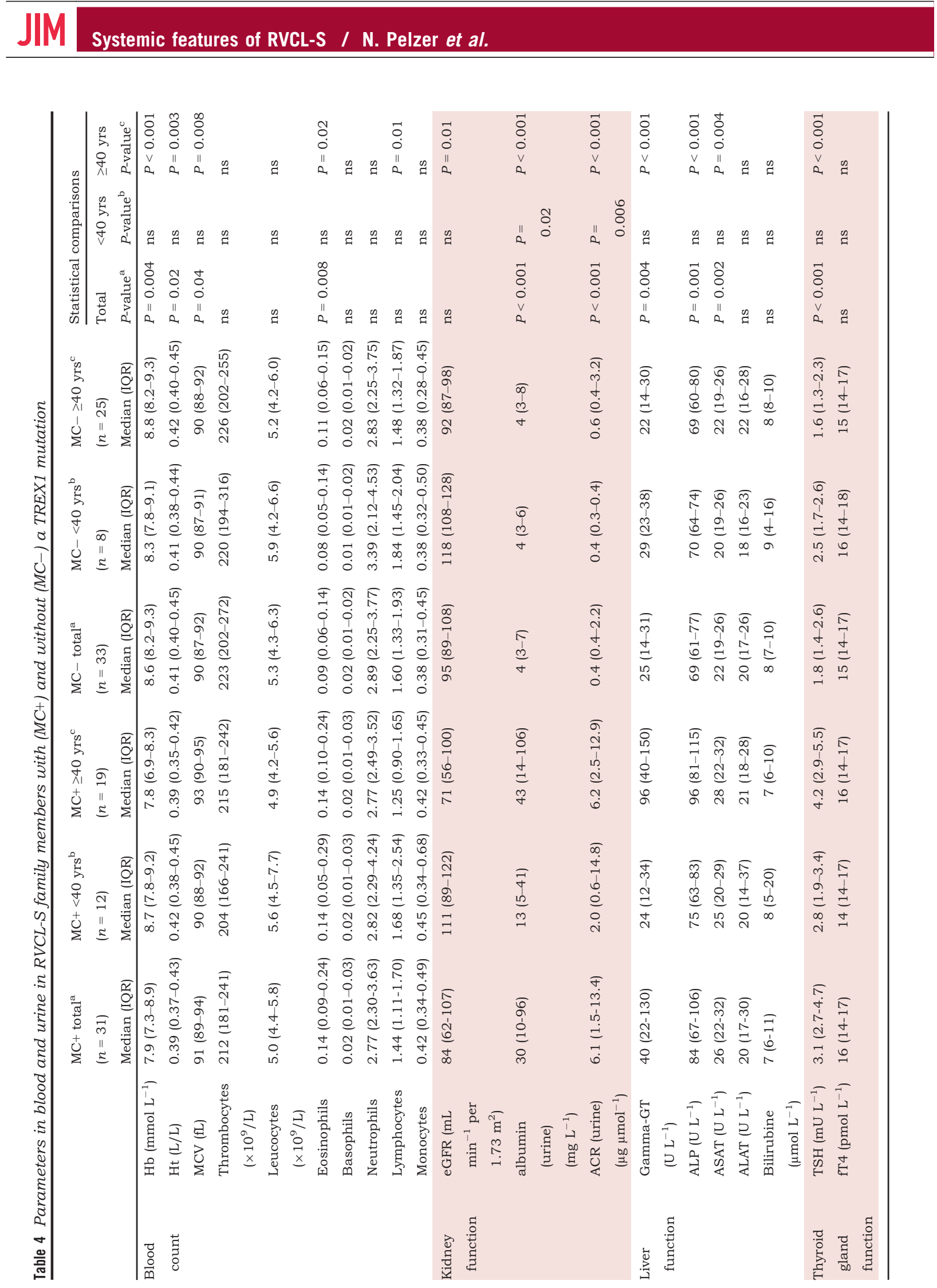


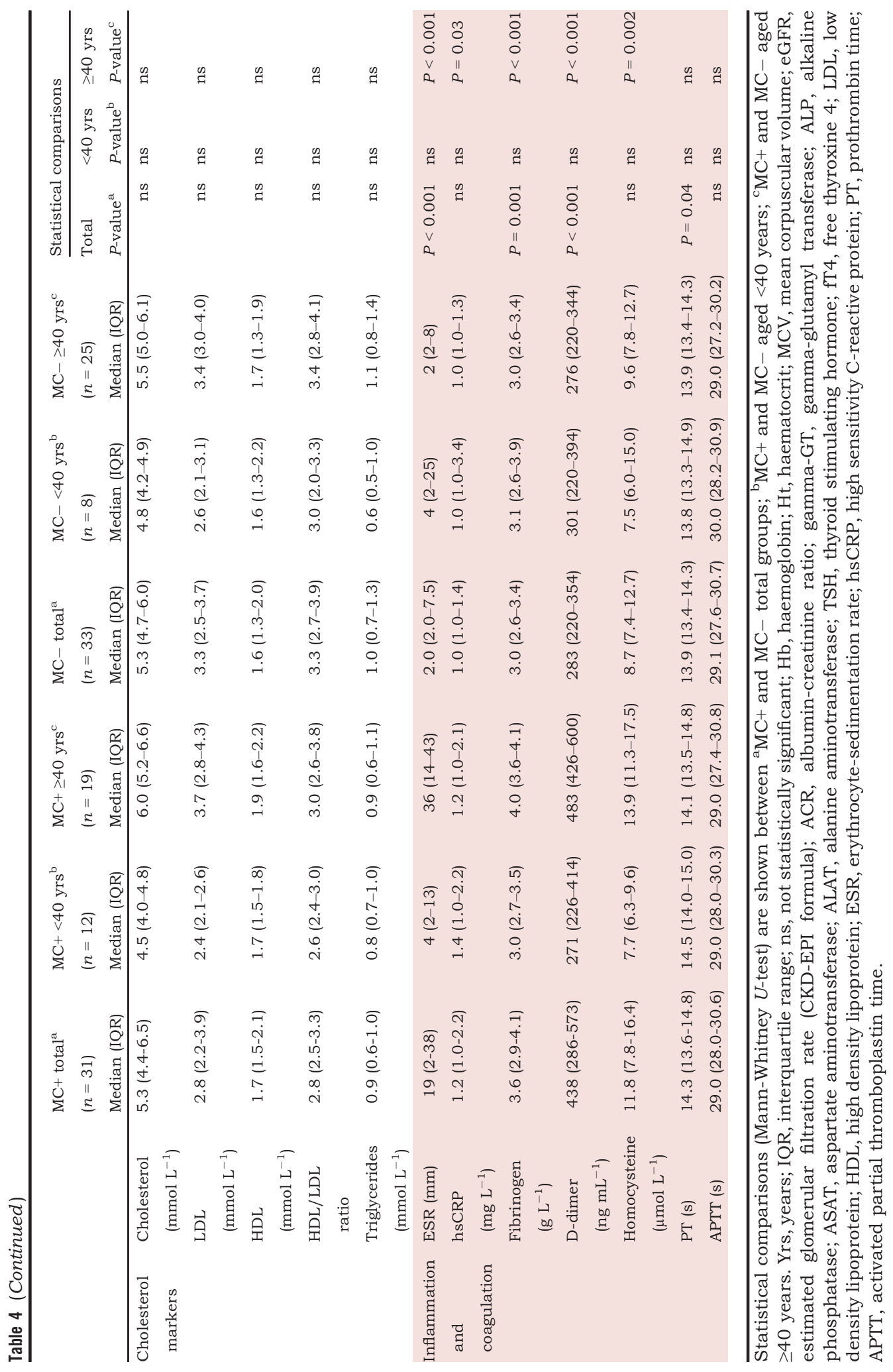




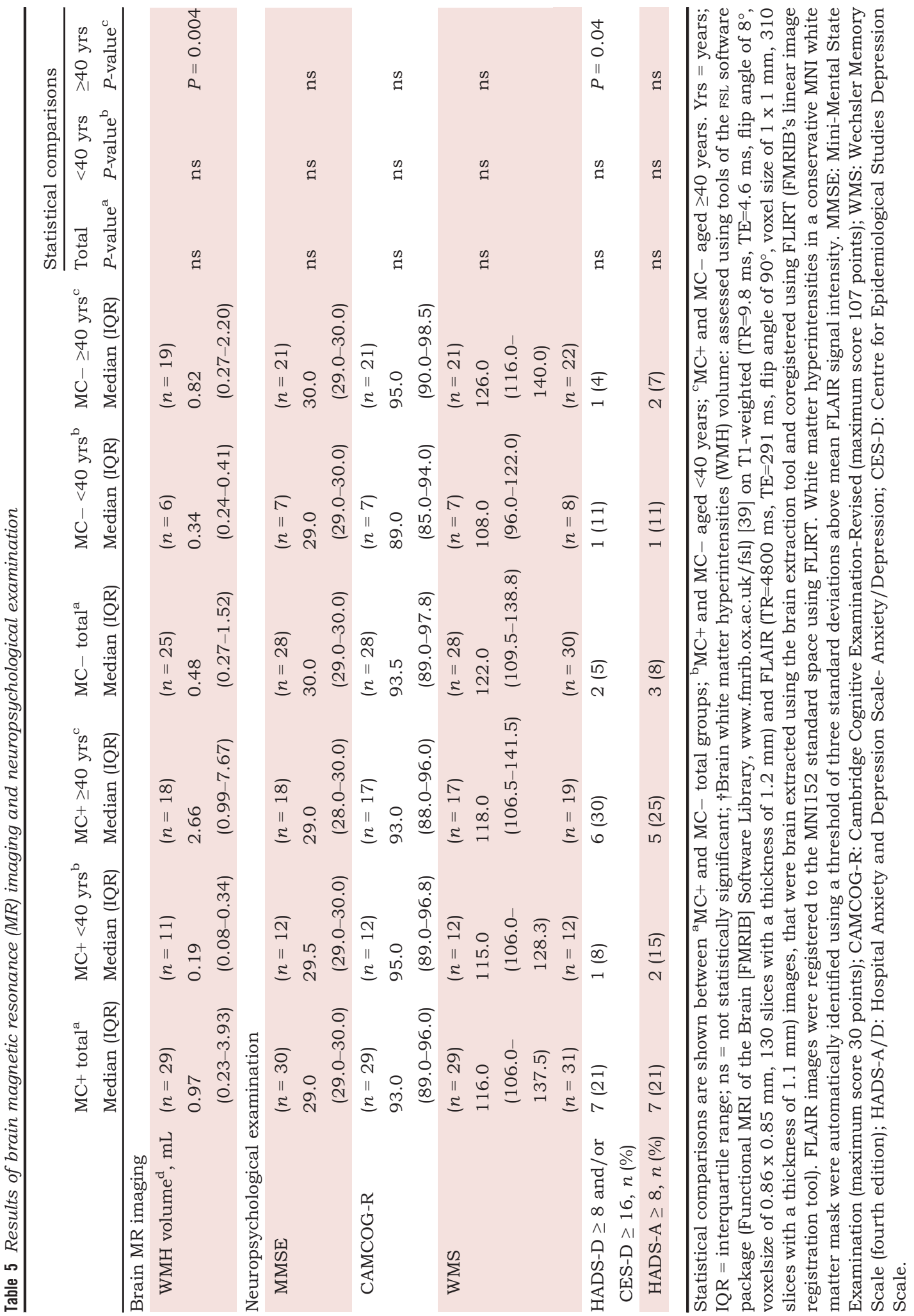


intracerebral mass lesions in the past, new 'pseudotumours' were not detected.

\section{Discussion}

We conducted an extensive and detailed cross-sectional study into the clinical course and ophthalmological, cerebral and notably systemic manifestations of RVCL-S in $33 \mathrm{MC}+$ and $37 \mathrm{MC}-$ from three Dutch RVCL-S families. Typically, vascular retinopathy and Raynaud's phenomenon presented from age 20 onwards, followed by kidney disease from age 35, and liver disease, anaemia likely due to gastrointestinal bleeding, migraine, and subclinical hypothyroidism, from age 40. The characteristic clinical and neuroimaging manifestations of global and focal brain dysfunction started mildly around age 50, to progressively worsen and becoming fatal over the next 1015 years. We suggest to add anaemia, gastrointestinal bleeding, and subclinical hypothyroidism as supportive diagnostic criteria for RVCL-S (Tables 6 and 7).

The sample size of our study, although considerable in view of the low prevalence of the disease, is probably too small for an accurate detailed assessment of the full clinical spectrum of RVCL-S. Moreover, the cross-sectional design also precludes an accurate estimate of the exact disease course over four decades. On the other hand, the clinical pattern across families and individual patients appears rather consistent in the present and previous studies $[2,4,5]$. We therefore believe that, despite the above limitations, the clinical characteristics and tentative disease course we found in our study do seem to reflect the grand clinical picture of RVCL-S rather well. The gold standard, a detailed, prospective follow-up study over several decades in large numbers of $\mathrm{MC}+$ and families, seems rather impossible.

Other than microvasculopathy due to RVCL-S, we did not reveal any other good explanation for internal organ disease in RVCL-S. For instance, $\mathrm{MC}+$ with albuminuria did not have high blood pressure and those with liver disease did not have high alcohol intake. Gastrointestinal bleeding might explain anaemia in at least some patients [2], warranting endoscopic evaluation with gastroand colonoscopy and possibly targeted treatment. Large angiodysplasias might require treatment with endoscopic argon plasma coagulation. Kidney disease was mild (eGFR $>40 \mathrm{~mL} \mathrm{~min}^{-1}$ per
Table 6 Current diagnostic criteria for RVCL-S [2]

Major diagnostic criteria

Vascular retinopathy (which in the early phases is associated with retinal haemorrhages, intraretinal microvascular abnormalities, and/or cotton wool spots)

Features of focal and/or global brain dysfunction associated on MRI with (i) punctate T2 hyperintense white matter lesions with nodular enhancement; and/or (ii) larger T2 hyperintense white matter mass lesions with rim-enhancement, mass effect, and surrounding oedema

Family history of autosomal dominant inheritance with middle-age onset of disease manifestations ${ }^{a}$

Demonstration of a C-terminal frameshift mutation in

TREX1 to genetically confirm the diagnosis

Supportive features

On CT focal white matter calcifications and/or on MRI nonenhancing punctate T2 hyperintense white matter lesions at an age that non-specific age-related white matter hyperintensities are infrequent

Microvascular liver disease (nodular regenerative

hyperplasia)

Microvascular kidney disease (arterio- or

arteriolonephrosclerosis, glomerulosclerosis)

Possibly associated features

Anaemia consistent with blood loss and/or chronic disease

Microscopic gastrointestinal bleeding

Hypertension

Migraine with or without aura

Raynaud's phenomenon (typically mild)

$\overline{{ }^{a}}$ De novo mutations may be possible although none have been reported to date.

$1.73 \mathrm{~m}^{2}$ ) and therefore not considered a likely explanation for anaemia, except perhaps in one MC+ who had an eGFR of $21 \mathrm{~mL} \mathrm{~min}^{-1}$ per $1.73 \mathrm{~m}^{2}$ and accordingly was treated with darbepoetin.

Seven $\mathrm{MC}+$ in our study proved to have subclinical hypothyroidism, which is a novel finding in RVCL$\mathrm{S}$. We did not find any other good explanation in these MC+, including use of medication [22]. Screening of thyroid function has not been routinely done in RVCL-S [23]. We are aware of only one report of hypothyroidism in RVCL-S, a 44- 
Table 7 Proposed new diagnostic criteria for RVCL-S.

Major diagnostic criteria
Vascular retinopathy (which in the early phases is
associated with retinal haemorrhages, intraretinal
microvascular abnormalities, and/or cotton wool spots)
Features of focal and/or global brain dysfunction
associated on MRI with (i) punctate T2 hyperintense
white matter lesions with nodular enhancement; and/or
(ii) larger T2 hyperintense white matter mass lesions
with rim-enhancement, mass effect, and surrounding
oedema

Family history of autosomal dominant inheritance with middle-age onset of disease manifestations ${ }^{a}$

Demonstration of a C-terminal frameshift mutation in

TREX1 to genetically confirm the diagnosis

Supportive features

On CT focal white matter calcifications and/or on MRI nonenhancing punctate T2 hyperintense white matter lesions at an age that non-specific age-related white matter hyperintensities are infrequent

Microvascular liver disease (nodular regenerative hyperplasia)

Microvascular kidney disease (arterio- or

arteriolonephrosclerosis, glomerulosclerosis)

Anaemia consistent with blood loss and/or chronic disease $^{\mathrm{b}}$

Microscopic gastrointestinal bleeding ${ }^{\mathrm{b}}$

Subclinical hypothyroidism ${ }^{\mathrm{C}}$

Possibly associated features

Raynaud's phenomenon (typically mild)

Migraine with or without aura (typically relatively late

onset)

Hypertension

${ }^{\mathrm{a}}$ De novo mutations may be possible although none have been reported to date.

${ }^{\mathrm{b}}$ Moved from possibly associated to supportive features.

${ }^{\mathrm{c}} \mathrm{New}$ supportive criterion

year-old male, but the association with RVCL-S was considered fortuitous [24]. Of interest, hypothyroidism has been reported in 14 patients with Aicardi-Goutières syndrome, which can be caused by homozygous missense mutations in TREX1 [25], that is different to the heterozygous C-terminal truncating mutations causing RVCL-S. Subclinical hypothyroidism is also considered a marker of endothelial dysfunction in diabetic retinopathy and chronic kidney disease [26, 27]. Subclinical hypothyroidism is a common disorder, with an estimated prevalence of $6 \%$ in the general population, which may increase to $17 \%$ in the elderly, most prominently in females [28, 29]. The prevalence of subclinical hypothyroidism in $\mathrm{MC}-$ over age 40 is $8 \%(2 / 25)$, and thus comparable to the prevalence in the general population, the prevalence in $\mathrm{MC}+$ over age 40 is markedly increased with $37 \%(7 / 19)$ compared to the general population.

We found an increased prevalence of Raynaud's phenomenon in RVCL-S (42\%), in line with previous reports [2, 30]. Prevalence figures were higher (both in $\mathrm{MC}+$ and $\mathrm{MC}-$ ) when using Miller's criteria $[4,7]$ than when using the recent international consensus criteria [8] and greatly exceeded the prevalence figures of around 5\% usually found in the general population [31]. As a positive family history of Raynaud's phenomenon increases the odds of developing the condition nearly 17 -fold [31], additional genetic risk factors seem to play an important role as well.

Lifetime prevalence of migraine was lower (27\%) than previously reported (59\%) [2]. This might have been due to that $13 / 33=39 \%$ of the newly identified $\mathrm{MC}+$ in our study was younger than 40 years, the usual age at onset of migraine in RVCL-S. Many of these $\mathrm{MC}+$ might thus still develop migraine later on in life. In the general population, migraine typically starts before age 25 [32]. Why migraine begins so much later in RVCL-S is unknown but one could envisage that migraine in RVCL-S is a secondary phenomenon due to progressive vasculopathy.

Neurological manifestations were associated with higher white matter hyperintensity volume on MRI and often remained mild and unnoticed. Cerebral mass lesions usually present around age 55 and were, without exception, associated with major neurological deficits [2]. Depressive symptoms were more common among $\mathrm{MC}+$. There was no evidence of major cognitive impairment in our cohort of relatively young patients, but cognitive decline was present in previously described large cohorts of RVCL-S patients [2]. Long-term follow-up data are warranted to get more insight in the exact age at onset of the symptomatology of RVCL-S.

How RVCL-S TREX1 mutations lead to multiple organ disease is unknown. Most organs affected in 
RVCL-S heavily rely on an intact endothelial barrier to maintain normal function. We found pharmacological [33] and biochemical evidence for endothelial dysfunction in RVCL-S [33, 34]. Patients with RVCL-S had abnormal increased circulating levels of the markers of endothelial function angiopoietin-2 and Von Willebrand Factor antigen and propeptide [32]. Neuropathological studies revealed fibrinoid vascular necrosis or thickened hyalinized vessels, and multilaminated basement membranes on ultrastructural images of both the brain and internal organs, suggesting endothelial cell damage [2, 5, 35, 36]. Altogether, this seems to suggest that RVCL-S is a systemic endotheliopathy.

The underlying mechanisms for the putative endothelial dysfunction are also unknown. Atherosclerosis does not seem to play a major role as vascular risk factors were no more common among $\mathrm{MC}+$ than in $\mathrm{MC}-$. Autoimmune mechanisms have been implicated. TREX1 is a DNA exonuclease that may prevent autoimmune activation by selfDNA [37]. RVCL-S frameshift mutations result in a truncated $\mathrm{C}$-terminus leaving the enzymatic activity of the N-terminal truncated TREX1 protein intact. TREX1 escapes nonsense-mediated decay, usually seen with truncations, as it is a one-exon gene. Lack of the C-terminal part of the TREX 1 protein results in dysregulation of oligosaccharyltransferase activity of the endoplasmic reticulum, which in turn leads to aberrant glycosylation and production of free glycans that may trigger the autoimmune response [38]. Inflammatory mechanisms might also be involved as we found increased levels of inflammatory markers (i.e. ESR, hsCRP, fibrinogen, D-dimer and homocysteine) in older $\mathrm{MC}+$.

In summary, RVCL-S is a rare, fatal, and probably underdiagnosed systemic small vessel disease, clinically typically starting around age 20 with progressive blindness due to vascular retinopathy and Raynaud's phenomenon. From age 35-40 onwards, most patients will develop multiple internal organ disease, justifying regular screening, followed a decade later by progressive characteristic and ultimately fatal cerebral deficits. Worldwide, only 16 unrelated families with confirmed RVCL-S have been described, of which three originate from and live in The Netherlands [2]. As this is a small country with only 17 million inhabitants, this striking observation does seem to suggest that many families and patients with RVCL-S remain, globally, unidentified.

\section{Conflict of interest statement}

No conflict of interests to declare.

\section{Financial disclosure statement}

N. Pelzer, E.S. Hoogeveen, J. Haan, R. Bunnik, C.C. Poot, E.W. van Zwet, A. Inderson, A.J. Fogteloo, M.E.J. Reinders, H.A.M. Middelkoop, M.C. Kruit reports no disclosures, A.M.J.M. van den Maagdenberg reports consultancy support from Novartis and independent support from European Community. M.D. Ferrari reports grants and consultancy or industry support from Medtronic, Novartis, Amgen, Lilly and TEVA and independent support from the European Community, NWO, NIH and the Dutch Heart \& Brain Foundations. G.M. Terwindt reports independent support from NWO, European Community, the Dutch Heart Foundation, and the Dutch Brain Foundation.

\section{Funding acknowledgement}

This work was supported by grants of the Netherlands Organization for Scientific Research (NWO) [VIDI 91711319 to G.M.T.] and the European Community (EC) [FP7-EUROHEADPAIN - no. 602633 to M.D.F. \& A.v.d.M.; FP7-NIMBL - no. 241779 to A.v.d.M.]. The funding agencies had no role in the design or conduct of the study.

\section{References}

1 Richards A, van den Maagdenberg AM, Jen JC et al. Cterminal truncations in human $3^{\prime}-5^{\prime}$ DNA exonuclease TREX1 cause autosomal dominant retinal vasculopathy with cerebral leukodystrophy. Nat Genet 2007; 39: 1068-70.

2 Stam AH, Kothari PH, Shaikh A et al. Retinal vasculopathy with cerebral leukoencephalopathy and systemic manifestations. Brain 2016; 139: 2909-22.

3 Grand MG, Kaine J, Fulling K et al. Cerebroretinal vasculopathy. A new hereditary syndrome. Ophthalmology 1988; 95: $649-59$.

4 Terwindt GM, Haan J, Ophoff RA et al. Clinical and genetic analysis of a large Dutch family with autosomal dominant vascular retinopathy, migraine and Raynaud's phenomenon. Brain 1998; 121: 303-16.

5 Jen J, Cohen AH, Yue Q et al. Hereditary endotheliopathy with retinopathy, nephropathy, and stroke (HERNS). Neurology 1997; 49: 1322-30.

6 DiFrancesco JC, Novara F, Zuffardi O et al. TREX1 Cterminal frameshift mutations in the systemic variant of retinal vasculopathy with cerebral leukodystrophy. Neurol Sci 2015; 36: 323-30.

7 Miller D, Waters DD, Warnica W, Szlachcic J, Kreeft J, Theroux P. Is variant angina the coronary manifestation of a 
generalized vasospastic disorder? N Engl J Med 1981; 304: 763-6.

8 Maverakis E, Patel F, Kronenberg DG et al. International consensus criteria for the diagnosis of Raynaud's phenomenon. $J$ Autoimmun 2014; 48-49: 60-5.

9 Headache Classification Committee of the International Headache Society (IHS) The International Classification of Headache Disorders, 3rd edition. Cephalalgia 2018; 38: $1-211$.

10 van Oosterhout WP, Weller CM, Stam AH et al. Validation of the web-based LUMINA questionnaire for recruiting large cohorts of migraineurs. Cephalalgia 2011; 31: 1359-67.

11 Bjelland I, Dahl AA, Haug TT, Neckelmann D. The validity of the Hospital Anxiety and Depression Scale. An updated literature review. J Psychosom Res 2002; 52: 69-77.

12 Sawyer Radloff L. The CES-D Scale: A Self-Report Depression Scale for Research in the General Population. Appl Psych Meas 1977; 3: 385-401.

13 Louter MA, Bosker JE, van Oosterhout WP et al. Cutaneous allodynia as a predictor of migraine chronification. Brain 2013; 136: 3489-96.

14 Chobanian AV, Bakris GL, Black HR et al. Seventh report of the Joint National Committee on Prevention, Detection, Evaluation, and Treatment of High Blood Pressure. Hypertension 2003; 42: 1206-52.

15 Mahoney FI, Barthel DW. Functional evaluation: the Barthel index. Md State Med J 1965; 14: 61-5.

16 van Swieten JC, Koudstaal PJ, Visser MC, Schouten HJ, van Gijn J. Interobserver agreement for the assessment of handicap in stroke patients. Stroke 1988; 19: 604-7.

17 Bouman Z, Hendriks MP, Kerkmeer MC, Kessels RP, Aldenkamp AP. Confirmatory Factor Analysis of the Dutch Version of the Wechsler Memory Scale-Fourth Edition (WMS-IV-NL). Arch Clin Neuropsychol 2015; 30: 228-35.

18 Huppert FA, Brayne C, Gill C, Paykel ES, Beardsall L. CAMCOG-a concise neuropsychological test to assist dementia diagnosis: socio-demographic determinants in an elderly population sample. Br J Clin Psychol 1995; 34: 529-41.

19 Folstein MF, Folstein SE, McHugh PR. "Mini-mental state". A practical method for grading the cognitive state of patients for the clinician. J Psychiatr Res 1975; 12: 189-98.

20 Winkler DT, Lyrer P, Probst A et al. Hereditary systemic angiopathy (HSA) with cerebral calcifications, retinopathy, progressive nephropathy, and hepatopathy. J Neurol 2008; 255: $77-88$.

21 Vodopivec I, Oakley DH, Perugino CA, Venna N, HedleyWhyte ET, Stone JH. A 44-year-old man with eye, kidney, and brain dysfunction. Ann Neurol 2016; 79: 507-19.

22 Barbesino G. Drugs affecting thyroid function. Thyroid 2010; 20: $763-70$.

23 Gutmann DH, Fischbeck KH, Sergott RC. Hereditary retinal vasculopathy with cerebral white matter lesions. Am J Med Genet 1989; 34: 217-20.

24 Mateen FJ, Krecke K, Younge BR et al. Evolution of a tumorlike lesion in cerebroretinal vasculopathy and TREX1 mutation. Neurology 2010; 75: 1211-3.

25 Crow YJ, Chase DS, Lowenstein SJ et al. Characterization of human disease phenotypes associated with mutations in TREX1, RNASEH2A, RNASEH2B, RNASEH2C, SAMHD1, ADAR, and IFIH1. Am J Med Genet A 2015; 167A: 296-312.
$26 \mathrm{Wu}$ J, Yue S, Geng J et al. Relationship between Diabetic Retinopathy and Subclinical Hypothyroidism: a meta-analysis. Sci Rep 2015; 5: 12212 .

27 Afsar B, Yilmaz MI, Siriopol D et al. Thyroid function and cardiovascular events in chronic kidney disease patients. $J$ Nephrol 2017; 30: 235-42.

28 Wiersinga WM. Subclinical hypothyroidism and hyperthyroidism. I. Prevalence and clinical relevance. Neth $J$ Med 1995; 46: 197-204.

29 Hennessey JV, Espaillat R. Subclinical hypothyroidism: a historical view and shifting prevalence. Int $J$ Clin Pract 2015; 69: $771-82$.

30 Hottenga JJ, Vanmolkot KR, Kors EE et al. The 3p21.1-p21.3 hereditary vascular retinopathy locus increases the risk for Raynaud's phenomenon and migraine. Cephalalgia 2005; 25: 1168-72.

31 Garner R, Kumari R, Lanyon P, Doherty M, Zhang W. Prevalence, risk factors and associations of primary Raynaud's phenomenon: systematic review and meta-analysis of observational studies. BMJ Open 2015; 5: e006389.

32 Stewart WF, Wood C, Reed ML, Roy J, Lipton RB. Cumulative lifetime migraine incidence in women and men. Cephalalgia 2008; 28: $1170-8$.

33 de Boer I, Stam AH, Buntinx L et al. RVCL-S and CADASIL display distinct impaired vascular function. Neurology 2018; 91: e956-e963.

34 Pelzer N, Bijkerk R, Reinders MEJ et al. Circulating endothelial markers in retinal vasculopathy with cerebral leukoencephalopathy and systemic manifestations. Stroke 2017; 48: 3301-7.

35 Kolar GR, Kothari PH, Khanlou N, Jen JC, Schmidt RE, Vinters HV. Neuropathology and genetics of cerebroretinal vasculopathies. Brain Pathol 2014; 24: 510-8.

36 Tsubata Y, Morita T, Morioka T et al. Renal histopathological findings of retinal vasculopathy with cerebral leukodystrophy. CEN Case Rep 2018; 7: 83-9.

37 Yang YG, Lindahl T, Barnes DE. Trex 1 exonuclease degrades ssDNA to prevent chronic checkpoint activation and autoimmune disease. Cell 2007; 131: 873-86.

38 Hasan M, Fermaintt CS, Gao $\mathrm{N}$ et al. Cytosolic nuclease TREX1 regulates oligosaccharyltransferase activity independent of nuclease activity to suppress immune activation. Immunity 2015; 43: 463-74.

39 Jenkinson M, Beckmann CF, Behrens TE, Woolrich MW, Smith SM. FSL. NeuroImage 2012; 62: 782-90.

Correspondence: Gisela M. Terwindt, Department of Neurology, Leiden University Medical Centre, Albinusdreef 2, PO Box 9600, 2300 RC Leiden, The Netherlands.

(fax: 0031715248253; e-mail: g.m.terwindt@lumc.nl).

\section{Supporting Information}

Additional Supporting Information may be found in the online version of this article:

Figure S1. Lack of association between albumin urine concentration versus systolic and diastolic blood pressure measured in visit 2 . 